\title{
Applications of the decomposition methods to some nonlinear partial differential equations
}

\author{
Hami Gündoğdu, Ömer Faruk Gözükızıl \\ Sakarya University, Department of Mathematics, Sakarya, Turkey
}

Received: 6 September 2017, Accepted: 11 december 2017

Published online: 22 July 2018.

\begin{abstract}
In this paper, we consider the inhomogeneous Benjamin-Bona-Mahony-Peregrine-Burgers (BBMPB) equation with its initial condition. The decomposition methods such as Adomian and Laplace are applied to this equation. Then, the solution satisfying the given initial condition is gained by using each method. To demonstrate these decomposition methods are how effective and convenient for solving the nonlinear partial differential equations(NLPDEs), solutions of some NLPDEs subjected to initial conditions are attained by these method. In the application of the method, it is clearly noticed that there is no need to convert the nonlinear terms into the linear ones due to the Adomian polynomials. In addition to this simplicity, it is seen that each method gives the same solution with a fast convergence by taking the advantage of the noise terms.
\end{abstract}

Keywords: Adomian and Laplace Decomposition Methods, the noise term, Adomian polynomials, Benjamin Bona Mahony Peregrine Burgers equation.

\section{Introduction}

In almost all fields of science and engineering, most problems can be represented by linear or nonlinear partial differential equations (shortly, N/LPDEs). To proceed in these fields, solutions to this kind of problems should be obtained. Important information about the encountered problems can be achieved by getting solutions to these type of equations. So, it is of a very significance role in modern science and engineering. In this regard, numerous researches have been made to find ways to obtain the solutions of NLPDEs. Eventually, many analytical and numerical methods have been established and used to reach this goal. Some of the methods are; the perturbation method [1-5], the homotopy perturbation method [3-7], the delta perturbative method [8], the Modified Decomposition method [9-16], the Adomian Decomposition method [15-22], the Laplace Decomposition method [15, 16, 22-27].

Firstly, let us consider the Benjamin-Bona-Mahony-Burgers (BBMB) [28] given by

$$
u_{t}-u_{x x t}-\alpha u_{x x}+\gamma u_{x}+f(u)_{x}=0
$$

Here, $u(x, t)$ is speed of liquid flowing in horizontal $\mathrm{x}$ direction, $\alpha$ is a positive constant, $\gamma$ is arbitrary real constant, and $f(u)$ is a smooth nonlinear function in $C^{2}$. When $f(u)_{x}=u u_{x}$ with $\alpha=0$ and $\beta=1$, equation (1) turns into the alternative regularized long-wase equation proposed by Peregrine [29] and Benjamin [30]. This equation characterizes a balance between nonlinear and dispersive effects, yet it does not take place in calculation of dissipation. In the perspective of physics, equation (1) with the dissipative term $\alpha u_{x x}$ is suggested when the good predictive power is wished. This kind of problem emerges in both the bore propagation and the water waves. Now, let us consider the Benjamin-Bona-Mahony

\footnotetext{
* Corresponding author e-mail: hamigundogdu@ sakarya.edu.tr
} 
equations given by

$$
u_{t}-\alpha u_{x x}+u_{x}+f(u)_{x}=0
$$

As it is known that the dispersive effect of the equation (1) is same with the equation (2). Because of this fact, equation (1) is called the Benjamin-Boan-Mahony-Burgers (BBMB) equations. But, it is an interesting situation that it is not proposed by Benjamin or Mahanoy or Burgers, check [31]. These equations, (1) and (2), take a large place in the literature, see [32-34]. So, they have been studied in different points of view. Here, let us give some works related to these equations. In [32], new kinds of solitary wave solutions to the equation (1) by a variable coefficient balancing act method. In [33], equation (1) is considered with $f(u)_{x}=u^{2}$. In this work, the extended tanh method is applied to it and gained the solitary wave solutions. In [34], Kaya takes the equation (1) with $f(u)_{x}=u^{p}$, for any positive $p$. The solitary wave solutions are obtained by using the Adomian decomposition method. In this equation, if we take $\theta, \beta \neq 0$ and $f(u)_{x}=\theta u u_{x}+\beta u_{x x x}$ then the BBMB equation takes the form

$$
u_{t}-u_{x x t}-\alpha u_{x x}+\gamma u_{x}+\theta u u_{x}+\beta u_{x x x}=0
$$

which is suggested Peregrine, Benjamin, et al[30]. This equation is called Benjamin-Bona-Mahony-Peregrine-Burgers. In this study, we consider BBMPB equation of the form

$$
u_{t}-u_{x x t}-\alpha u_{x x}+\gamma u_{x}+\theta u u_{x}+\beta u_{x x x}=x+\gamma t+\theta x t^{2},
$$

with initial condition $u(x, 0)=0$.

The purpose of this work is to show that the Laplace and Adomian decomposition methods are usefull in solving NLPDEs subject to the initial conditions. Therefore, we apply these decomposition methods to the inhomogeneous BBMPB equation and some NLPDEs with their initial conditions. The applications of these methods point out that these methods are completely suitable for getting the solutions of NLPDEs with initial conditions.

This study is arranged as follows. In section 2, descriptions of the methods are given in detail. In section 3, ADM and LDM are applied to BBMPB equation given in (4) and some other NLPDEs, and the solutions which satisfy the given initial conditions. As a result of this work, some conclusions are given in section 4.

\section{Outline of the methods}

\subsection{The Laplace decomposition method}

This method is given as in the following manner. First, let us consider the nonlinear partial differential equation in operator form

$$
D u(x, t)+R u(x, t)+N u(x, t)=h(x, t)
$$

with initial conditions $u(x, 0)=f(x)$ and $u_{t}(x, 0)=g(x)$. Here, $D$ is a second order partial differential operator w.r.t $t, R$ is a remaining linear operator, $N$ represents a general nonlinear differantial operator, $h(x, t)$ is a source term.

Firstly, the laplace transform is applied to both sides of (5). Then, we have

$$
L[D u(x, t)]+L[R u(x, t)]+L[N u(x, t)]=L[h(x, t)] .
$$


By the differentiation property of the Laplace transform, we get

$$
L[u(x, t)]=\frac{f(x)}{s}+\frac{g(x)}{s^{2}}+\frac{1}{s^{2}} L[h(x, t)]-\frac{1}{s^{2}}[L[R u(x, t)+L[N u(x, t)]]]
$$

where $s$ is Laplace domain function. In this method, $u(x, t)$ is defined as

$$
u(x, t)=\sum_{n=0}^{\infty} u_{n} .
$$

The nonlinear terms, $N$, given by infinite series

$$
N(u(x, t))=\sum_{n=0}^{\infty} A_{n}(u)
$$

where $A_{n}$ represents the Adomian polynomials. Adomian polynomials are defined as

$$
A_{n}=\frac{1}{n !} \frac{d^{n}}{d \alpha^{n}}\left[N\left(\sum_{n=0}^{\infty} \alpha^{n} u_{n}\right], \quad n=0,1,2, \ldots\right.
$$

By using this definition, the first few terms can be given as follows:

$$
\begin{gathered}
A_{0}=N\left(u_{0}\right), \\
A_{1}=u_{1} N^{\prime}\left(u_{0}\right), \\
A_{2}=u_{2} N^{\prime}\left(u_{0}\right)+\frac{1}{2 !} u_{1}^{2} N^{\prime \prime}\left(u_{0}\right),
\end{gathered}
$$

and other terms can be derived in a similar way. Now, substituting (8) and (9) into equation (7) gives us

$$
L\left(\sum_{n=0}^{\infty} u_{n}\right)=\frac{f(x)}{s}+\frac{g(x)}{s^{2}}+\frac{1}{s^{2}} L[h(x, t)]-\frac{1}{s^{2}} L\left[R u_{n}(x, t)\right]-\frac{1}{s^{2}} L\left(\sum_{n=0}^{\infty} A_{n}(u)\right) .
$$

Linearity of laplace transform converts it into the following form:

$$
\sum_{n=0}^{\infty} L\left(u_{n}\right)=\frac{f(x)}{s}+\frac{g(x)}{s^{2}}+\frac{1}{s^{2}} L[h(x, t)]-\frac{1}{s^{2}} L\left[R u_{n}(x, t)\right]-\frac{1}{s^{2}} \sum_{n=0}^{\infty}\left[L\left(A_{n}(u)\right)\right] .
$$

Comparing both sides of (15) yields the following equalities:

$$
\begin{gathered}
L\left(u_{0}\right)=\frac{f(x)}{s}+\frac{g(x)}{s^{2}}+\frac{1}{s^{2}} L[h(x, t)], \\
L\left(u_{1}\right)=-\frac{1}{s^{2}} L\left[R u_{0}(x, t)\right]-\frac{1}{s^{2}} \sum_{n=0}^{\infty}\left[L\left(A_{0}(u)\right)\right], \\
L\left(u_{2}\right)=-\frac{1}{s^{2}} L\left[R u_{1}(x, t)\right]-\frac{1}{s^{2}} \sum_{n=0}^{\infty}\left[L\left(A_{1}(u)\right)\right],
\end{gathered}
$$


and so on. In general, the recursive relation is given by

$$
L\left(u_{n+1}\right)=-\frac{1}{s^{2}} L\left[R u_{n}(x, t)\right]-\frac{1}{s^{2}} \sum_{n=0}^{\infty}\left[L\left(A_{n}(u)\right)\right], n \geq 0 .
$$

To get $u_{0}, u_{1}, \ldots, u_{n+1}$, we take the inverse laplace transform of this equation. Then, we obtain

$$
\begin{gathered}
u_{0}=f(x)+\operatorname{tg}(x)+H(x, t), \\
u_{n+1}=-L^{-1}\left[\frac{1}{s^{2}} L\left[R u_{n}(x, t)\right]-\frac{1}{s^{2}} \sum_{n=0}^{\infty}\left[L\left(A_{n}(u)\right)\right]\right]
\end{gathered}
$$

where $H(x, t)=L^{-1}\left[\frac{1}{s^{2}} L[h(x, t)]\right]$.

\subsection{The Adomian decomposition method}

Let us consider the nonlinear partial differential equation (5). It can be written as

$$
L_{t} u(x, t)+R u(x, t)+N u(x, t)=h(x, t)
$$

where $L_{t}=\frac{\partial}{\partial t^{2}}$. Firstly, let us rewrite the equation (22) as

$$
L_{t} u(x, t)=h(x, t)-R u(x, t)-N u(x, t) .
$$

Then, applying $L_{t}^{-1}$ to both sides of this equation and using the initial conditions gives us

$$
u(x, t)=f(x)+\operatorname{tg}(x)+L_{t}^{-1} h(x, t)-L_{t}^{-1} R u(x, t)-L_{t}^{-1} N u(x, t)
$$

where $L_{t}^{-1}=\int_{0}^{t} \int_{0}^{t}() d t d$.$t . For the linear terms, we use the decomposition series given by$

$$
u(x, t)=\sum_{n=0}^{\infty} u_{n}
$$

For the nonlinear terms, we use the following infinite series of Adomian polynomials given by

$$
N(u(x, t))=\sum_{n=0}^{\infty} A_{n}(u)
$$

where $A_{n}$ are Adomian polynomials. We obtain the recursive relation:

$$
\begin{gathered}
u_{0}(x, t)=f(x)+\operatorname{tg}(x)+L_{t}^{-1} h(x, t), \\
u_{k+1}(x, t)=-L_{t}^{-1}(R u(x, t))-L_{t}^{-1}\left(A_{k}\right) .
\end{gathered}
$$

This relation provides us with the components of the solution $u(x, t)$. The first few terms of solution are given as

$$
u_{0}(x, t)=f(x)+\operatorname{tg}(x)+L_{t}^{-1} h(x, t)
$$




$$
\begin{aligned}
& u_{1}(x, t)=-L_{t}^{-1} R u_{0}(x, t)-L_{t}^{-1}\left(A_{0}\right) \\
& u_{2}(x, t)=-L_{t}^{-1} R u_{1}(x, t)-L_{t}^{-1}\left(A_{1}\right) .
\end{aligned}
$$

In both methods, the solution $u(x, t)$ is given by

$$
u(x, t)=\sum_{n=0}^{\infty} u_{n}=u_{0}+u_{1}+u_{2}+\ldots u_{n}+\ldots
$$

Putting these components in $u(x, t)=\sum_{n=0}^{\infty} u_{n}$ and using the noise terms phenomena gives us the solution rapidly.

\section{Application of the methods}

The methods are applied to the BBMPB equation and some other inhomogenous NLPDEs with initial conditions.

\subsection{Solving BBMPB equation by $L D M$}

In this section, LDM is applied to BBMPB equation given by (4). Taking the laplace transform of both sides, we get

$$
L\left[u_{t}\right]=L\left[u_{x x t}\right]+\alpha L\left[u_{x x}\right]-\gamma L\left[u_{x}\right]-\beta L\left[u_{x x x}\right]+L\left[x+\gamma t-\theta x t^{2}\right]-L[N(u)]
$$

where $N(u)=u u_{x}$. Using the properties of laplace transform gives

$$
L[u]=\frac{1}{s}\left(L\left[u_{x x t}\right]+\alpha L\left[u_{x x}\right]-\gamma L\left[u_{x}\right]-\beta L\left[u_{x x x}\right]+L\left[x+\gamma t-\theta x t^{2}\right]-L[N(u)]\right) .
$$

So, we have the following recursive relation:

$$
\begin{gathered}
L\left[u_{0}\right]=\frac{1}{s} L\left[x+t \gamma+x t^{2} \theta\right), \\
L\left[u_{k+1}\right]=\frac{1}{s} L\left[u_{k_{x x t}}\right]+\alpha \frac{1}{s} L\left[u_{k_{x x}}\right]-\gamma \frac{1}{s} L\left[u_{k_{x}}\right]-\beta \frac{1}{s} L\left[u_{k_{x x x}}\right]-\theta \frac{1}{s} L\left[A_{k}\right] .
\end{gathered}
$$

Suppose that the inverse of laplace transform exists. Then taking the inverse of laplace transform, we get

$$
\begin{gathered}
u_{0}=x t+\frac{\gamma}{2} t^{2}+\frac{\theta}{3} x t^{3}, \\
u_{1}=-\frac{\gamma}{2} t^{2}-\frac{7 \gamma \theta}{24} t^{4}-\frac{\gamma \theta^{2}}{18} t^{6}-\frac{\theta}{3} x t^{3}-\frac{4 \theta^{2}}{15} x t^{5}-\frac{4 \theta^{3}}{63} x t^{2},
\end{gathered}
$$

and so on for the other components. By canceling the noise terms, $\frac{\gamma}{2} t^{2}$ and $\frac{\theta}{3} x t^{3}$, from the component $u_{0}$ gives us the solution:

$$
u(x, t)=x t
$$




\subsection{Solving BBMPB equation by $A D M$}

Firstly, let us write the equation (4) as

$$
L_{t}(u)=u_{x x t}+u_{x x} \alpha-u_{x} \gamma-u u_{x} \theta-u_{x x x} \beta+x+t \gamma+x t^{2} \theta, u(x, 0)=0,
$$

where $L_{t}$ is a first order derivative w.r.t $t$. Then, we get

$$
u=L_{t}^{-1}\left(u_{x x t}+\alpha u_{x x}-\gamma u_{x}-\beta u_{x x x}\right)+L_{t}^{-1}\left(x+\gamma t+\theta x t^{2}\right)-L_{t}^{-1} N(u),
$$

where $L_{t}^{-1}=\int_{0}^{t}() d$.$t . Using the decomposition series for the linear terms and Adomian polynomial for the nonlinear$ terms gives the following relation

$$
\begin{gathered}
u_{0}=L_{t}^{-1}\left(x+\gamma t+\theta x t^{2}\right) \\
u_{1}=L_{t}^{-1}\left(u_{0_{x x t}}+\alpha u_{0_{x x}}-\gamma u_{0_{x}}-\beta u_{0_{x x x}}\right)-L_{t}^{-1}\left(A_{0}\right)
\end{gathered}
$$

and so on for the other components. Here, $A_{0}(u)=u_{0} u_{0_{x}}$. Then, we obtain

$$
\begin{gathered}
u_{0}=x t+\frac{\gamma}{2} t^{2}+\frac{\theta}{3} x t^{3}, \\
u_{1}=-\frac{\gamma}{2} t^{2}-\frac{7 \gamma \theta}{24} t^{4}-\frac{\gamma \theta^{2}}{18} t^{6}-\frac{\theta}{3} x t^{3}-\frac{4 \theta^{2}}{15} x t^{5}-\frac{4 \theta^{3}}{63} x t^{2} .
\end{gathered}
$$

So, the noise terms are $\frac{\gamma}{2} t^{2}$ and $\frac{\theta}{3} x t^{3}$. Canceling these terms from the component $u_{0}$ gives the solution

$$
u_{0}=x t
$$

\subsection{Examples}

In this part, the LDM and ADM are applied to some inhomogeneous NLPDEs with their initial values.

Example 1. Consider the following equation

$$
u_{t t}-u_{x x}+u_{x x t}-2 u_{x t t}-u_{x} u_{t}=\sin (t)-\cos (t)
$$

with the initial conditions $u(x, 0)=x+1$ and $u_{t}(x, 0)=0$.

(a) Let us apply the LDM to this equation. Then, we have

$$
L\left[u_{t t}\right]=L\left[u_{x x}\right]-L\left[u_{x x t}\right]+L\left[2 u_{x t t}\right]+L[N(u)]+L[\sin (t)-\cos (t)]
$$

where $N(u)=u_{x} u_{t}$. The differentiation property of the laplace transform and initial conditions give

$$
L[u]=\frac{x+1}{s}+\frac{0}{s^{2}}+\frac{1}{s^{2}} L[\sin (t)-\cos (t)]+\frac{1}{s^{2}}\left(L\left[u_{x x}\right]-L\left[u_{x x t}\right]+L\left[2 u_{x t t}\right]\right)+\frac{1}{s^{2}}(L[N(u)]) .
$$

Then, we get the following recursive relation:

$$
L\left[u_{0}\right]=\frac{x+1}{s}+\frac{1}{s^{2}} L[\sin (t)-\cos (t)]
$$




$$
L\left[u_{k+1}\right]=\frac{1}{s^{2}}\left(L\left[u_{x x}\right]-L\left[u_{x x t}\right]+L\left[2 u_{x t t}\right]\right)+\frac{1}{s^{2}}(L[N(u)]) .
$$

Assume that the inverse of laplace transform exists. Then taking the inverse of laplace transform, we obtain

$$
\begin{gathered}
u_{0}=x+t+\cos (t)-\sin (t), \\
u_{1}=-1-t+\frac{t^{2}}{2}+\cos (t)+\sin (t) .
\end{gathered}
$$

Here, we have the noise terms, $t$ and $\sin (t)$. Removing the noise terms from $u_{0}$ provides us with the solution

$$
u(x, t)=x+\cos (t)
$$

(b) The ADM is applied to the equation (47). It can be rewritten as

$$
L_{t t}(u)=u_{x x}-u_{x x t}+2 u_{x t t}+u_{x} u_{t}+\sin (t)-\cos (t)
$$

where $L_{t t}$ is a second order derivative w.r.t $t$. Applying the inverse of $L_{t t}$ gives

$$
u=L_{t t}^{-1}\left(u_{x x}-u_{x x t}+2 u_{x t t}\right)+L_{t t}^{-1}\left(u_{x} u_{t}\right)+L_{t t}^{-1}(\sin (t)-\cos (t))
$$

where $L_{t} t^{-1}=\int_{0}^{t} \int_{0}^{t}() d t d$.$t . Using the decomposition series for the linear terms and Adomian polynomial for the nonlinear$ terms gives the following relation

$$
\begin{gathered}
u_{0}=x+1+L_{t} t^{-1}(\sin (t)-\cos (t)), \\
u_{1}=L_{t} t^{-1}\left(u_{x x}-u_{x x t}+2 u_{x t t}\right)+L_{t} t^{-1}\left(A_{0}(u)\right)
\end{gathered}
$$

where $A_{0}(u)=N(u)=u_{0_{x}} u_{0_{t}}$. Then, we get

$$
\begin{gathered}
u_{0}=x+t-\sin (t)+\cos (t), \\
u_{1}=-1-t+\frac{t^{2}}{2}+\cos (t)+\sin (t) .
\end{gathered}
$$

Clearly, the noise terms are $t$ and $\sin (t)$.If we remove these terms from $u_{0}$, we gain the solution as

$$
u(x, t)=x+\cos (t)
$$

Example 2. Consider the following equation

$$
u_{t}-u_{x x}+u_{x x t t}+\frac{1}{2} u_{x x} u_{t t}=2 x t-2 x t^{2}
$$

with the initial condition $u(x, 0)=-x^{2}$. 
(a) Let us apply the LDM to the equation (62). The laplace transform of both sides is taken. Then, we have

$$
L\left[u_{t}\right]=L\left[u_{x x}\right]-L\left[u_{x x t t}\right]-L[N(u)]+L\left[2 x t-2 x t^{2}\right]
$$

where $N(u)=\frac{1}{2} u_{x x} u_{t t}$. By using the differentiation property of the laplace transform and initial condition, we get

$$
L[u]=\frac{-x^{2}}{s}++\frac{1}{s} L\left[2 x t-2 x t^{2}\right]+\frac{1}{s}\left(L\left[u_{x x}\right]-L\left[u_{x x t t}\right]-\frac{1}{s}(L[N(u)])\right.
$$

Then, this equality gives the following recursive relation:

$$
\begin{gathered}
L\left[u_{0}\right]=\frac{-x^{2}}{s}+\frac{1}{s} L\left[2 x t-2 x t^{2}\right], \\
L\left[u_{k+1}\right]=\frac{1}{s}\left(L\left[u_{x x}\right]-L\left[u_{x x t}\right]-\frac{1}{s}(L[N(u)]) .\right.
\end{gathered}
$$

Assume that the inverse of laplace transform exists. If we take the inverse of laplace transform, then we get

$$
\begin{gathered}
u_{0}=-x^{2}+x t^{2}-2 x t+2 t, \\
u_{1}=-2 t+2 x t
\end{gathered}
$$

Comparing $u_{0}$ and $u_{1}$ gives the noise terms as $2 t$ and $-2 x t$. Removing the noise terms from $u_{0}$ provides us with the solution

$$
u(x, t)=x t^{2}-x^{2}
$$

(b) The ADM is applied to the equation (62). It can be rewritten as

$$
L_{t}(u)=u_{x x}-u_{x x t t}-\frac{1}{2} u_{x x} u_{t t}+2 x t-2 x t^{2}
$$

where $L_{t}$ is a first order derivative w.r.t $t$. By applying the inverse of $L_{t}$ to both sides, we get

$$
u=L_{t}^{-1}\left(u_{x x}-u_{x x t t}\right)+L_{t}^{-1}\left(\frac{1}{2} u_{x x} u_{t t}\right)+L_{t}^{-1}\left(2 x t-2 x t^{2}\right)
$$

where $L_{t}^{-1}=\int_{0}^{t} d t$. If we use the decomposition series for the linear terms and Adomian polynomial for the nonlinear terms, then we have the following relation:

$$
\begin{gathered}
u_{0}=-x^{2}+L_{t}^{-1}\left(2 x t-2 x t^{2}\right), \\
u_{1}=L_{t}^{-1}\left(u_{x x}-u_{x x t t}\right)+L_{t}^{-1}(N(u))
\end{gathered}
$$

where $A_{0}(u)=N(u)=\frac{1}{2} u_{x x} u_{t t}$. Then, we obtain

$$
u_{0}=2 t-2 x t+x t^{2}-x^{2}
$$




$$
u_{1}=-2 t-2 x t
$$

It is obvious that the noise terms are $2 t$ and $-2 x t$. If we remove these terms from $u_{0}$, we gain the solution as

$$
u(x, t)=x t^{2}-x^{2}
$$

\section{Conclusion}

In this study, we consider the BBMPB equation with its initial condition. To obtain the solution of this equation, ADM, and LDM are applied. Then, it is seen that the same exact solution is gained by both methods. Then, these methods are applied to some NLPDEs with initial conditions and the solutions are gained. In these applications, it is shown that these decomposition methods are powerful in solving nonlinear ones with initial and boundary conditions in addition to their effectiveness and usefulness in solving linear partial differential equations. Compared to other methods for solving nonlinear partial differential equations with initial conditions, there is no need for linearization of nonlinear terms thanks to the Adomian polynomials. Moreover, the solution can easily and rapidly be attained with the help of the noise terms.

\section{Competing interests}

The authors declare that they have no competing interests.

\section{Authors' contributions}

All authors have contributed to all parts of the article. All authors read and approved the final manuscript.

\section{References}

[1] Damil N., Potier-Ferry M., Najah A., Chari R., Lahmam H., An iterative method based upon Pade approximamants, Communications In Numerical Methods In Engineering, 15, (1999), 701-708.

[2] Liu G.-L., New research directions in singular perturbation theory: artificial parameter approach and inverse-perturbation technique, Proceeding of the 7th Conference of the Modern Mathematics and Mechanics, Shanghai, 61, (1997), 47-53.

[3] He J.-H., A coupling method of homotopy technique and perturbation technique for nonlinear problems, International Journal Of Non-Linear Mechanics, 35, (2000), 37-43.

[4] Cadou J.-M., Moustaghfir N., Mallil E. H., N. Damil, Potier-Ferry M., Linear iterative solvers based on pertubration techniques, Comptes Rendus Mathematique, 332, (2001), 457-462.

[5] Mallil E., Lahmam H., Damil N., Potier-Ferry M., An iterative process based on homotopy and perturbation techniques, Computer Methods In Applied Mechanics Engineering, 190, (2000), 1845-1858.

[6] He J.-H., An approximate solution technique depending upon artificial parameter, Communications In Nonlinear Science And Numerical Simulaton, 3, (1998), 92-97.

[7] He J.-H., Newton-like iteration method for solving algebraic equations, Communications In Nonlinear Science And Numerical Simulaton, 3, (1998), 106-109.

[8] Bender C. M., Pinsky K. S., Simmons L. M., A new perturbative approach to nonlinear problems, Journal Of Mathematical Physics, 30, (1989), 1447-1455.

[9] Adomian G., Rach R., Inhomogeneous nonlinear partial differential equations with variable coefficients, Applied Mathematics Letters, 5, (1992), 11-12. 
[10] Adomian G., Rach R., Modified decomposition solutions of nonlinear partial differential equations, Applied Mathematics Letters, 5, (1992), 29-30.

[11] Adomian G., Rach R., A modified decomposition series, Computers And Mathematacis with Applications, 23, (1992), 17-23.

[12] Adomian G., Rach R., Modified decomposition solution of linear and nonlinear boundary-value problems, Nonlinear Analysis, 23, (1994) 615-619.

[13] Mohammed M., Tarig M. E., A study some systems of nonlinear partial differential equations by using Adomian and modified decomposition methods, African Journal Of Mathematics And Computer Science Research, 7, (2014), 61-67.

[14] Hsu J. C., Lai H. Y., Chen C. K., An innovative eigenvalue problem solver for free vibration of uniform Timoshenko beams by using the Adomian modified decomposition method, Journal Of Sound And Vibration, 325, (2009), 451-470.

[15] Gündoğdu H., Gözükızıl Ö., Solving Nonlinear Partial Differential Equations by Using Adomian Decomposition Method, Modified Decomposition Method and Laplace Decomposition Method, MANAS Journal Of Engineering, 5, (2017), 1-13.

[16] Gündoğdu H., Gözükızıl Ö., Obtaining the solution of Benney-Luke Equation by Laplace and adomian decomposition methods, Sakarya University Journal Of Science, 21, (2017), 1524-1528.

[17] Adomian G., Nonlinear stochastic systems theory and applications to physics, in Mathematics And Its Applications, Kluwer Academic Publishers, 46, (1989), 10-224.

[18] Adomian G., Solving frontier problems of physics: the decomposition method, in Fundamental Theories Of Physics, Kluwer Academic Publishers-Plenum, Springer Netherlands, 60, (1994), 6-195.

[19] Adomian G., An analytic solution of the stochastic Navier-Stokes system, in Foundations Of Physics, Kluwer Academic Publisher, Springer Science And Business Media, 21, (1991), 831-843.

[20] Adomian G., R. Rach, Linear and nonlinear Schrï $\frac{1}{2}$ ndinger equations, in Foundations Of Physics, Kluwer Academic PublishersPlenum, 21, (1991), 983-991.

[21] Adomian G., Solution of physical problems by decomposition, Computers And Mathematacis with Applications, 27, (1994), 145154.

[22] Adomian G., Solution of nonlinear P.D.E, Applied Mathematics Letters, 11, (1998), 121-123.

[23] Khuri S. A., A laplace decomposition algorithm applied to class of nonlinear differential equations, Journal Of Mathematical Analysis And Applications, 1, (2001), 141-155.

[24] Majid K., Hussain M., Hossein J., Yasir K., Application of Laplace decomposition method to solve nonlinear coupled partial differential equations, World Applied Sciences Journal, 9, (2010), 13-19.

[25] Majid K., Muhammed A. G., Application of Laplace decomposition to solve nonlinear partial differential equations, International Journal Of Advanced Research In Computer Science, 2, (2010), 52-62.

[26] Hosseinzadeh H., Jafari H., Roohani M., Application of Laplace decomposition method for solving Klein-Gordon equation, World Applied Sciences Journal, 8, (2010), 809-813.

[27] Majid K., Hussain M., Application of Laplace decomposition method on semi-infinite domain, Numer. Algor., 56, (2011), $211-218$.

[28] Quintero J. R., Munoz Grajales J. C., Instability of solitary waves for a generalized Benney-Luke equation, Nonlinear Analysis Theory, Methods And Applications, 68, (2008), 3009-3033.

[29] D.H. Peregrine, Calculations of the development of an undular bore, J. Fluid Mech. 25 (1996) $321 ? 330$.

[30] T.B. Benjamin, J.L. Bona, J.J. Mahony, Model equations for long waves in nonlinear dispersive systems, Philos Trans R Soc London, Ser A, 272 (1972) 47-78.

[31] Peregrine D. H., Calculations of the development of an undular bore, J. Fluid Mech., 25, (1996), 321-330.

[32] Ming Mei, Large-time behavior of solution for generalized Benjamin?Bona?Mahony?Burgers Equations, Nonlinear Anal. 33 (1998) 699-714.

[33] Chen Yong, Li Biao, Zhang Hongqing, Exact solutions of two nonlinear wave equations with simulation terms of any order, Comm. Nonlinear Sci. Numer. Simulation 10 (2) (2005) 133-138.

[34] H. Zhang, G.M. Wei, Y.T. Gao, On the general form of the Benjamin?Bona?Mahony equation in fluid mechanics, Czech J. Phys. 52 (2002) 344-373.

[35] D. Kaya, A numerical simulation of solitary-wave solutions of the generalized regualized longwave equation, Appl. Math. Comput. 149 (2004) 833-841. 